\title{
EDITORIAL
}

\section{HEALTH DIGITALIZATION IS A NEED OF HOUR}

\author{
Taimoor Hassan ${ }^{1,2}$ \\ 'School of Pharmacy \& School of Medicine, Changzhou University, Jiangsu, China, \\ ${ }^{2}$ Department of Health Professional Technologies, The University of Lahore, Pakistan
}

Information system (IS) is the backbone of all organizations, programmes or projects. The swift spread of technology has also brought to the development and extension of the information systems utilized by the governments. In the case of Punjab province of Pakistan, there has been an exponential expansion of information systems by the public sector, particularly the health sector. ${ }^{1}$ In order to simplify the data flow and increase the performance of personnel, especially those operating in the remote fields, a resilient, fully integrated and complete digital medical and registry systems is a need of hour. Data accuracy will equip government to prepare one step ahead for the future, optimize present resources in line with need, reduce drug and supply pilfering, monitor staff performance efficiently and improve services quality. ${ }^{2}$ All of these will help directly in achieving the broader aim of universal healthcare. Once fully operational, the idea suggested would be a single, centered database, allowing people to login and have access to their own health records right from the start. In Punjab there are already several fragmented information systems. ${ }^{3}$

It is recommended that these technologies should be integrated with a single health reporting system such as digital medical records. This should be done in tandem with the registration of persons and families and then any additional information which include health amenities that individuals are provided with at any step of their life, is entered in real time. In addition, the system will have the capacity to record new births and mortalities and to provide modules to each framework in the government's health services provision model. ${ }^{4}$ At a later point, the private sector might also be involved in the same system. Incorporated into the same system will also be administrative, monitoring, logistical and human resources modules. Security of data and privacy should be guaranteed by proper legislations. ${ }^{1}$ In order to assure compliance with safety and privacy records of individuals, regulations like the US Health Insurance Portability and Accountability Act (HIPAA) may have to be enacted in the country. Similarly, it is also necessary to create a strategy for data encryption and develop databases for primary and secondary backups. The future of health records is a comprehensive in-house data entering and recording system in real-time and is suggested for implementation throughout the country. The whole process may take several years to complete but at every stage of information technology integration we must choose same directions i.e., for each type of service delivery in the health sector an integrated system with numerous modules. ${ }^{2,3}$

\section{Salient Points to be considered:}

1. Manual reports can contribute to delayed reporting and permit data manipulation for or under different services and illness occurrences reporting.

2. Real-time electronic reporting reduces the chances of data falsification and optimize timeliness. ${ }^{1}$

3. A single source of truth is the hallmark of a system of reporting in real-time, which provides data directly into the health database, i.e., electronic medical records, for persons seeking any medical treatment.

KEY WORDS: Computerized Medical Records Systems; Electronic Health Records; Health Information Systems. Cite as: Hassan T. Health digitalization is a need of hour [editorial]. Gomal J Med Sci 2021 Jan-Mar; 19(1):1-2. https://doi.org/10.46903/gjms/19.01.951

\section{Corresponding Author:}

Dr. Taimoor Hassan

Post-graduate Scholar

School of Pharmacy \& School of Medicine

Changzhou University, Jiangsu, China

E-mail: taimoorhassan408.th@gmail.com

Date Submitted: $\quad 25-09-2020$

Date Revised: $\quad 02-11-2020$

Date Accepted:
4. The electronic medical records system also helps to electronically monitor the quality and the volume of services provided and also further improves their connection with regular monitoring systems in real-time monitoring.

5. Day to day examination of situation and modifications may be made on the basis of evidence produced by the report. ${ }^{1,2}$ 


\section{REFERENCES}

1. Primary \& Secondary Healthcare Department Punjab. Punjab Health Survey-II (Progress on Key Health Indicators, 2017) [cited 2020 October 10]. Available at: https://pshealthpunjab.gov.pk/ Home/ProgressReports

2. Improving data use in decision making: An intervention to strengthen health systems-Measure Evaluation [Internet]. Measureevaluation. org. 2012 [cited 2020 October 10]. Available at: https://www.measureevaluation.org/resources/ publications/sr-12-73.html
3. Data quality audit tool-guidelines for implementation [Internet]. Measureevaluation.org. 2011 [cited 2020 October 10]. Available from: https://www.measureevaluation.org/resources/ publications/ms-08-29.html

4. AbouZahr C, Boerma T. Health information systems: the foundations of public health. Bull World Health Organ 2005;83(8):578-83.
CONFLICT OF INTEREST

Authors declare no conflict of interest. GRANT SUPPORT AND FINANCIAL DISCLOSURE None declared. 